\title{
Thoracic, abdominal and pelvic CT and US imaging features in children with hemophagocytic lymphohistocytosis
}

\author{
Hesham M AL-Shaalan ${ }^{1}$, Hanan M AL-Qahtani ${ }^{1}$, Riyadh N AL-Okaili ${ }^{1}$, Peter Cox ${ }^{2}$, Susanne \\ Benseler ${ }^{2}$, Mohamed Abdelhaleem², Sheila Weitzman ${ }^{2}$, Jeremy Friedman ${ }^{2}$, Rayfel Schneider ${ }^{2}$, \\ David Manson'2
}

1. National Guard Hospital, King Abdulaziz medical city/Riyadh, Saudi Arabia. 2. University of Toronto, Canada

Correspondence: Hanan M AL-Qahtani. Address: National Guard Hospital, King Abdulaziz medical city/Riyadh, Saudi Arabia. Email: dr-h.shaher@windowslive.com

Received: September 14, 2015 Accepted: November 1, 2015

URL: http://dx.doi.org/10.5430/ijdi.v3n1p34

\section{Abstract}

Background: Hemophagocytic lymphohistiocytosis/macrophage activation syndrome (HLH/MAS) is a life threatening condition characterized by uncontrolled autoimmune hyperinflammation based on various inherited or acquired immune deficiencies. Main symptoms and signs include fever, neurological symptoms, hepatosplenomegaly and cytopenia. This study aims to describe the chest, abdomen and pelvis CT findings as well as the US findings of the abdomen and pelvis, in a large series of patients diagnosed with HLH in a single institution.

Methods: This is a retrospective case review of the US and CT imaging features of the chest, abdomen and pelvis in 39 bone marrow biopsy-proven patients diagnosed with HLH/MAS that has been approved by our institutional review board (IRB). The study was done at one institution, The Hospital for Sick Children, Toronto, Canada. The imaging data of the 39 patients were retrieved using the PAC system. Two patients lacked available studies in the hospital records. The chest CT (26 patients), abdominal and pelvic CT (25 patients) and abdominal and pelvic US (34 patients) examinations in 37 children at presentation were retrospectively reviewed blindly by 2 pediatric radiologists. Discrepancy was resolved by consensus. Statistical analysis utilizing logistic regression and odds ratio have been used to estimate the patient's outcome.

Results: The major CT findings in the chest included pleural effusion in 16/26 patients (61.5\%), lymphadenopathy in $15 / 26$ patients (57.7\%) and air space disease in 12/26 patients (46.2\%). In the abdomen and pelvis, the major CT findings were as follows: ascites in 17/25 patients (68\%), hepatomegaly in 16/25 patients (64\%), splenomegaly in 14/25 patients (56\%), abdominal wall edema and periportal edema in 13/25 patients (52\%). Regarding ultrasound, hepatomegaly and splenomegaly were observed in 23/34 patients (67.6\%), ascites and echogenic kidneys were seen in 19/34 patients (55.9\%), and gall bladder wall thickening was found in 18/34 patients (52.9\%). A logistic regression model and odds ratio was used. We found that abdominal wall edema detected on CT has 8 times the risk of having a severe clinical outcome.

Conclusion: Large series of children with HLH/MAS demonstrated nonspecific imaging findings; however, in the appropriate clinical setting in acutely ill child, these findings should suggest the diagnosis of HLH/MAS to the radiologist. Early diagnosis and early management of this disorder can be life-saving.

\section{Keywords}

Hemophagocytic lymphohistiocytosis, Children, Imaging features 


\section{I ntroduction}

Hemophagocytic lymphohistiocytosis (HLH) is a life-threatening condition characterized by uncontrolled hyperinflammation based on various inherited or acquired immune deficiencies. Main symptoms and signs include fever, neurological symptoms, hepatosplenomegaly and cytopenia. There are two types of HLH/MAS, primary and secondary types. Primary HLH/MAS occurs in familial forms (FHLH) in which it is associated with the immune deficiencies. Chédiak-Higashi syndrome (CHS), Griscelli syndrome (GS) and X-linked lymphoproliferative syndrome (XLP) in which secondary HLH could occurs sporadically. Secondary HLH/MAS have unknown underlying immune deficiency and usually occurs with underlying systemic illness such as rheumatic diseases and malignancies. Both primary and secondary forms are triggered by infections. The diagnostic criteria for HLH include familial disease/known genetic defect and clinical/laboratory criteria (5/8 criteria) ${ }^{[1,2]}$. Biochemical markers include elevated triglyceride and ferritin, high levels of interleukin-2 receptor and low fibrinogen levels. Impaired function of natural killer (NK) cells and cytotoxic T-cells (CTL) is characteristic of all forms of HLH.

HLH/MAS in general is ineffective immune response that threatens the patient's life and may lead to death unless arrested by appropriate treatment ${ }^{[3]}$.

\section{Methods}

This is a retrospective case review of the US and CT imaging features of the chest, abdomen and pelvis in 39 bone marrow biopsy-proven patients diagnosed with HLH/MAS that has been approved by our IRB. This is the largest series of patients from a single institution compared with the individual series reported in the literature.

Our inclusion criteria were patients who had CT and/or US of the chest and/or abdomen and/or pelvis during their admission at presentation and who received the diagnosis of HLH/MAS by bone marrow biopsy. Patients who had no imaging studies at our institution (2 patients) or those who had imaging of parts of the body other than CT/US of the chest, abdomen and pelvis were excluded.

All available imaging studies of 37 children who met the inclusion criteria were retrieved using the PAC system. The chest CT (26 patients), abdominal and pelvic CT (25 patients) and abdominal and pelvic US (34 patients) examinations in the 37 children at presentation were retrospectively blindly reviewed by 2 pediatric radiologists. The CT and US findings were recorded on a data collection form. Differences in interpretations were resolved by consensus. After completion of the data base collection, the statistical significance of each modality (CT/US) and its corresponding imaging features was analyzed. The collected data were stratified according to the following: 1) primary underlying diagnosis, 2) incidence of imaging findings and 3) clinical severity at presentation (for example, ICU admission, hypotension, or shock). Logistic regression and odds ratio have been used to determine the mortality risk and clinical outcome.

\section{Results}

In our cohort group, there was slight female predominance (21 females/16 males) with a M: F ratio of 1:1.3. The patients were divided into 4 groups according to age (< 1 year, 5 patients; $1-5$ years, 11 patients; 5-10 years, 6 patients; and 10-17 years, 15 patients).

The clinical severity of the disease was broadly divided according to mild (floor admission) and severe (ICU/PICU admission) groups. Twenty-two patients were found to have mild clinical presentation, and 15 patients had severe clinical presentation. Regarding the clinical outcome of the disease, 29 patients were discharged to their home after treatment, whereas 8 patients unfortunately died 7 were females (mean age, 6.3 years) and 1 was male (age 6.7 years). The underlying disease of the patients who died were: 2 patients found to have EBV as an underlying cause. The remaining 6 patients had 
lymphoma, primary HLH/MAS, SLE, leukemia, lymphoproliferative disease and systemic fungal infection. The worst clinical outcome occurred in the third group (5-10 years) in which 4 of 6 patients died, followed by the first group ( $<1$ year) in which 2 of 5 patients died. In the second group (1-5 years), 1 of 11 died. The most favorable clinical outcome occurred in the last group (10-17 years) in which 1 of 15 patients died (see Table 1).

Table 1. Clinical severity and outcome in each age group

\begin{tabular}{lllllll}
\hline Age & \% of Clinically Mild & \% of Clinically Severe & \% of $\mathbf{M}$ & \% of $\mathbf{F}$ & \% of Death & \% of Discharge \\
\hline$<$ 1 year & $(3 / 5) 60$ & $(2 / 5) 40$ & $(3 / 5) 60$ & $(2 / 5) 40$ & $(2 / 5) 40$ & $(3 / 5) 60$ \\
1-5 years & $(8 / 11) 72.7$ & $(3 / 11) 27.3$ & $(6 / 11) 54.5$ & $(5 / 11) 45.5$ & $(1 / 11) 9.1$ & $(10 / 11) 90.9$ \\
5-10 years & $(2 / 6) 33.3$ & $(4 / 6) 66.7$ & $(2 / 6) 33.3$ & $(4 / 6) 66.7$ & $(4 / 6) 66.7$ & $(2 / 6) 33.3$ \\
10-17 years & $(9 / 15) 60$ & $(6 / 15) 40$ & $(5 / 15) 33.3$ & $(10 / 15) 66.7$ & $(1 / 15) 6.7$ & $(14 / 15) 93.3$ \\
\hline
\end{tabular}

Primary HLH/MAS was the most common underlying disease and was found in 7 patients (18.9\%). JRA was the second most common primary underlying disease (6 patients, 16.2\%). EBV and SLE were found in 4 patients each (10.8\%). Lymphoma and leukemia were the primary underlying disease in 3 patients each (8.1\%). Kawasaki disease was present in 2 patients (5.4\%). The other types of primary underlying disease associated with HLH/MAS in our cohort (LCH, varicella, CMV, encephalitis, lymphoproliferative disease, autoimmune disease, fungemia and lymphoma complicated with EBV infection) were observed in 1 patient each (2.7\%). The highest mortality was observed with EBV infection for which 2 of 4 patients died, both females with a mean age of 9.65 years (see Table 2). From imaging findings prospective, 25 patients had CT of the chest/abdomen and pelvis, and 1 patient had CT of the chest only (see Figures 1, 2).

Table 2. Clinical severity and outcome for each underlying disease

\begin{tabular}{lllllllll}
\hline Disease & No. of patients & Clinical severity (Mild/Severe) & Clinical outcome & Sex & & Age mean (yrs) \\
\hline Lymphoma & 3 & 2 & 1 & 2 & 1 & 2 & 1 & 9 \\
1HLH & 7 & 3 & 4 & 6 & 1 & 3 & 4 & 3.8 \\
JRA & 6 & 5 & 1 & 6 & 0 & 1 & 5 & 9.35 \\
EBV & 4 & 2 & 2 & 2 & 2 & 0 & 4 & 9.65 \\
SLE & 4 & 2 & 1 & 2 & 1 & 1 & 2 & 1.39 \\
Leukemia & 3 & 1 & 3 & 3 & 1 & 1 & 3 & 14.27 \\
Kawasaki & 2 & 2 & 0 & 2 & 0 & 2 & 0 & 5.4 \\
LCH & 1 & 1 & 0 & 1 & 0 & 1 & 0 & 0.44 \\
Varicella & 1 & 1 & 0 & 1 & 0 & 1 & 0 & 10.35 \\
CMV & 1 & 1 & 0 & 1 & 0 & 1 & 0 & 1.37 \\
LPD & 1 & 0 & 1 & 0 & 1 & 1 & 0 & 6.78 \\
Encephalitis & 1 & 0 & 1 & 1 & 0 & 0 & 1 & 16.3 \\
Fungemia & 1 & 0 & 1 & 0 & 1 & 0 & 1 & 0.66 \\
Autoimmune & 1 & 1 & 0 & 1 & 0 & 1 & 0 & 16.09 \\
Lymphoma/EB & 1 & 1 & 0 & 1 & 0 & 1 & 0 & 11.93 \\
\hline
\end{tabular}

The main CT findings on the chest in those 26 patients were as follows: pleural effusion (16/26 patients, 61.5\%), lymphadenopathy (15/26 patients, 57.7\%) and air space disease (12/26 patients, 46.2\%). Pericardial effusion was observed in $8 / 26$ patients (30.8\%). Pulmonary edema was noticed in 6/26 patients $(23.1 \%)$. Other findings such as pulmonary nodules (2/26, 7.7\%) and ground glass opacities (1/26, 3.8\%) were also detected.

Twenty-five patients had abdomen and pelvis CT scan. The most common CT findings in these patients were as follow: ascites (17/25 patients, 68\%), hepatomegaly (16/25 patients, 64\%), splenomegaly (14/25 patients, 56\%) and periportal/abdominal wall edema (13/25 patients each, 52\%). Lymphadenopathy and gall bladder wall thickening 
were observed in 12/25 patients each (48\%). Other findings included bowel wall thickening (8/25 patients, 32\%), pericholecystic fluid (4/25 patients, 16\%) and mesenteric edema (2/25 patients, $8 \%$ ).

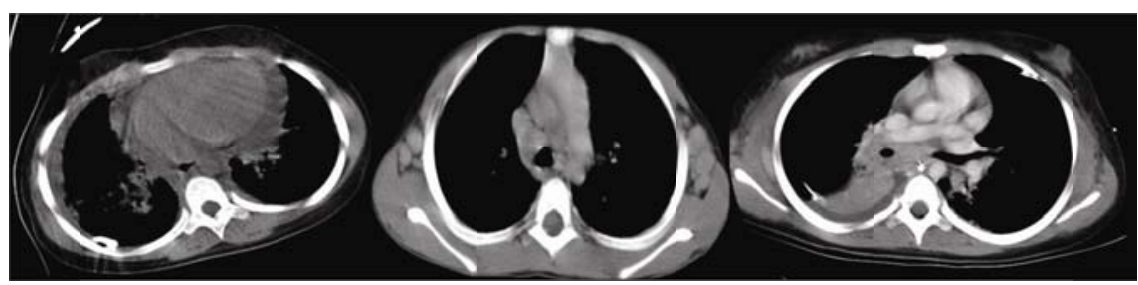

Figure 1. Chest CT in different patients with HLH/MAS showing pericardial effusion, lymphadenopathy and air space disease with pleural effusion

Figure 2. CT of the abdomen and pelvis in patients with HLH showing abdominal wall edema, periportal edema, ascites and bowel wall thickening

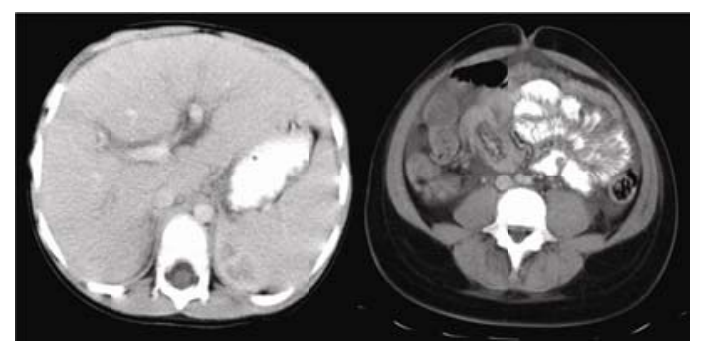

Regarding ultrasound, 34 patients had US of the abdomen and pelvis (see Figure 3). The most frequent ultrasound findings were as follows: hepatosplenomegaly (23/34 patients, 67.6\%), ascites and echogenic kidneys (19/34 patients each, 55.9\%) and gall bladder wall thickening (18/34 patients, 52.9\%). We also observed other findings in these patients, including periportal edema (13/34, 38.2\%), pleural effusion (12/34, 35.3\%), echogenic portal triads (10/34, 29.4\%), lymphadenopathy (9/34, 26.5\%), gall stones or sludge (6/34, 17.6\%) and bowel wall thickening and nephromegaly (5/34 each, $14.7 \%$ ). Incidental findings of either splenic or liver lesions were noticed in 2 patients (5.9\%). The size of the spleen and kidneys was determined using age-matched charts. The liver size was judged subjectively by identifying the inferior tip of the right lobe below the inferior pole of the right kidney.

Figure 3. Ultrasound of the abdomen in patients with HLH showing GB wall thickening, echogenic kidney and echogenic periportal triad

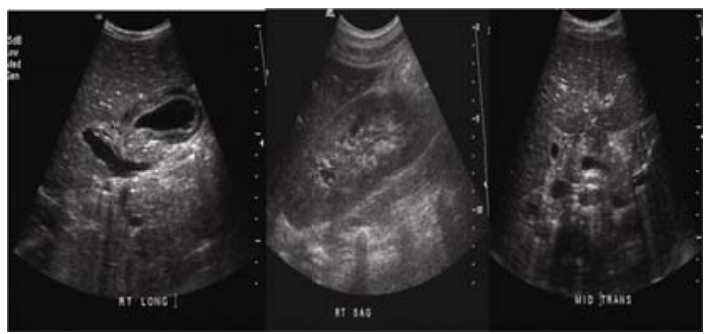

A logistic regression model was used. We found that abdominal wall edema detected on CT is a marker for severe clinical outcome with an odds ratio of 7.999, indicating that a patient with abdominal wall edema has 8 times the risk of having a severe clinical outcome.

\section{Discussion}

HLH/MAS is a life threating condition which is associated with high morbidity and mortality. Most patients affected by HLH/MAS have underlying causes, including rheumatologic, infectious and immunologic diseases. Patients with HLH/MAS can be divided into two groups: primary and secondary HLH/MAS patients. Primary HLH/MAS is an inherited, invariably fatal disease that occurs in approximately 1 in 50,000 births ${ }^{[4]}$. Patients with primary HLH/MAS have a median survival rate of 10 months and $10 \%$ survival after age $3^{[5]}$. 
Treatment of primary HLH/MAS consists of bone marrow transplantation, which can be curative ${ }^{[6]}$. Secondary HLH/MAS occurs in various diseases such as infections, neoplasia, hematological conditions and rheumatologic disorders ${ }^{[7]}$. In addition to disease processes, therapeutic regimens such as immunosuppression therapy have also been reported as a cause of secondary HLH/MAS. Other triggers of HLH/MAS include non-histiocytic malignancies, disorders of the immune system such as systemic lupus and Still’s disease, and Kawasaki disease ${ }^{[8]}$.

Imaging features in children with HLH/MAS are non-specific. Abdominal ultrasonography (US) is more likely to be requested at the time of presentation when the differential diagnosis is still broad. A report of six children with HLH/MAS (two children with primary HLH and four children with secondary HLH) emphasized the US findings of gallbladder wall thickening, increased periportal echogenicity, and lymphadenopathy in the porta hepatis; hepatosplenomegaly and ascites were also described ${ }^{[9]}$. Another report described two children with Epstein-Barr virus-associated HLH and imaging findings of gallbladder wall thickening, hepatosplenomegaly, ascites, pleural effusion, pulmonary edema, and cardiomegaly ${ }^{[10]}$.

More recently, 25 North American children with HLH/MAS were reviewed and revealed similar US findings $19^{[11]}$. These findings were supported by another study that reviewed the US features in 9 patients with HLH/MAS ${ }^{[12]}$.

These studies and their observations supported our findings in 34 abdominal and pelvic ultrasound examinations. In our larger group of patients, these findings were consistent and reproducible, suggesting their clinical significance, although they remain non- specific. We also observed a few other findings such as bowel wall thickening that could be explained by the systemic third spacing and fluid leaking that are observed in patients with HLH/MAS. Gall stones and biliary sludge were also observed, both of which are of unknown importance and are likely related to the severity of the patient's underlying clinical condition. Most of the changes observed by ultrasound were also observed on CT. There are few, if any, reports that have reviewed the CT imaging features in patients with HLH/MAS.A single case report described MRI findings of a child with acute lymphoblastic leukemia who developed HLH of which showed multiple rounded signal voids in the spleen and, to a lesser extent, in the liver that corresponded to hemosiderin deposits ${ }^{[13]}$.

We classified the CT and US imaging findings in our group into 3 groups based on their incidence rates. Group 1 represents findings with the lowest incidence rates; on CT (4\%-23\%), they include ground glass opacities, pulmonary nodules, mesenteric edema, pericholecystic fluid and pulmonary edema. On US (6\%-21\%), they include splenic/liver lesions, bowel wall thickening, nephromegaly and gall stone/sludge. Group 2 represents findings with intermediate incidence rates; on CT (31\%-52\%), they include pericardial effusion, bowel wall thickening, air space disease, lymphadenopathy, GB wall thickening, periportal edema and abdominal wall edema. On US (26\%-53\%), this group of findings includes lymphadenopathy, echogenic periportal triads, pleural effusion, periportal edema and GB wall thickening. Group 3 represents findings with the highest incidence rates. The CT findings (56\%-68\%) in this group include splenomegaly, lymphadenopathy, pleural effusion, hepatomegaly and ascites. On US (56\%-68\%), the findings in this group include ascites, echogenic kidneys, hepatomegaly and splenomegaly.

The imaging findings on CT and US were stratified based on clinical outcome. To determine whether any of these findings could predict the patients' clinical outcome, a logistic regression model was used. We found that abdominal wall edema detected on CT is a marker for severe clinical outcome with an odds ratio of 7.999, indicating that a patient with abdominal wall edema has 8 times the risk of having a sever clinical outcome. Conversely, splenomegaly in both CT and US was found to be a marker of mild clinical outcome with an odds ratio of 0.184 . These observations were consistent on both CT and US.

Although treatment strategies have been developed using the HLH-94 protocol ${ }^{[4]}$. The major problem lies in the early identification of these patients to expedite treatment initiation. A delay in the definitive diagnosis of HLH/MAS can be life threatening. Alternate diagnoses such as malignancy or septic shock may result in inappropriate treatment. Furthermore, the delay in diagnosis prevents optimal and timely treatment. 
Treatment at present for secondary HLH/MAS includes chemotherapeutic and immunosuppressive drugs, including corticosteroids, cyclosporine, and intravenous gammaglobulin ${ }^{[14,2,15]}$. Treatment is currently standardized by the HLH 94 protocol, which is a consensus by the HLH international group. The problem with the protocol is that not all patients respond as desired, and certain patients are treated with modified or additional medications.

Our study possessed limitations. It was retrospective, and radiologists were not blinded to the diagnosis and radiological reports, which could introduce a degree of subjective bias.

\section{Conclusion}

US or CT is more likely to be performed early in the diagnostic work-up of children with appropriate clinical setting suggestive of HLH/MAS. These findings are non-specific and could be observed in various other conditions such as hepatitis, infectious mononucleosis, EBV, leukemia, and LCH. This study in this relatively large group of children with HLH demonstrates a series of findings that, when present in the appropriate clinical setting of the acutely ill child, should suggest the diagnosis of HLH/MAS to the radiologist. Early diagnosis and acute management of this disorder can be life-saving.

\section{References}

[1] Grom AA. Macrophage activation syndrome and reactive hemophagocytic lymphohistiocytosis: the same entities? Curr Opin Rheumatol. 2003; 15: 587-590. PMid:12960485 http://dx.doi.org/10.1097/00002281-200309000-00011

[2] Ravelli A. Macrophage activation syndrome. Curr Opin Rheumatol. 2002; 14: 548-552. PMid:12192253 http://dx.doi.org/10.1097/00002281-200209000-00012

[3] Gritta Janka, Udo zur Stadt. Familial and Acquired Hemophagocytic Lymphohistiocytosis. Hematology. 2005; $2005:$ 82-88. PMid:16304363 http://dx.doi.org/10.1182/asheducation-2005.1.82

[4] Henter JI. Biology and treatment of familial hemophagocytic lymphohistiocytosis: importance of perforin in lymphocyte mediated cytotoxicity and triggering of apoptosis. Med Pediatr Oncol. 2002; 38: 305-309. PMid:11979453 http://dx.doi.org/10.1002/mpo.1340

[5] Arico M, Danesino C, Pende D, et al. Pathogenesis of haemophagocytic lymphohistiocytosis. Br J Haematol. $2001 ; 114: 761-769$. PMid:11564062 http://dx.doi.org/10.1046/j.1365-2141.2001.02936.x

[6] Fischer A, Cerf-Bensussan N, Blanche S, et al. Allogeneic bone marrow transplantation for erythrophagocytic lymphohistiocytosis. J Pediatr. 1986; 108: 267-270. http://dx.doi.org/10.1016/S0022-3476(86)81002-2

[7] Sawhney S, Woo P, Murray KJ. Macrophage activation syndrome: a potentially fatal complication of rheumatic disorders. Arch Dis Child. 2001; 85: 421-426. PMid:11668110 http://dx.doi.org/10.1136/adc.85.5.421

[8] Muise A, Tallett SE, Silverman ED. Are children with Kawasaki disease and prolonged fever at risk for macrophage activation syndrome? Pediatrics. 2003; 112: e495. PMid:14654653 http://dx.doi.org/10.1542/peds.112.6.e495

[9] Chateil J, Brun M, Perel Y, et al. Abdominal ultrasound findings in children with hemophagocytic lymphohistiocytosis. Eur Radiol. 1999; 9: 474-477. PMid:10087119 http://dx.doi.org/10.1007/s003300050695

[10] Moritani T, Aihara T, Oguma E, et al. Spectrum of Epstein-Barr virus infection in Japanese children: a pictorial essay. Clin Imaging. 2001; 25: 1-8. http://dx.doi.org/10.1016/S0899-7071(01)00257-1

[11] Fitzgerald NE, MacClain KL. Imaging characteristics of hemophagocytic lymphohistiocytosis. Pediatr Radiol. 2003 ; 33: $392-401$. PMid:12768255 http://dx.doi.org/10.1007/s00247-003-0894-9

[12] Schmidt MH, Sung L, Shuckett BM. Hemophagocytic lymphohistiocytosis in children: abdominal US findings within 1 week of presentation. Radiology. 2004; 230: 685-689. PMid:14990835 http://dx.doi.org/10.1148/radiol.2303030223

[13] Zilkha A, Madan V, Leonidas JC, et al. Liver and spleen MRI findings in virus-associated hemophagocytic syndrome in a patient with acute lymphocytic leukemia. Pediatr Radiol. 1998; 28: 920-922. PMid:9880630 http://dx.doi.org/10.1007/s002470050498

[14] Ramanan AV, Schneider R. Macrophage activation syndrome-what's in a name. J Rheumatol. 2003; 30: $2513-2516$. PMid:14719185

[15] Imashuku S. Clinical features and treatment strategies of Epstein-Barr virus-associated hemophagocytic lymphohistiocytosis. Crit Rev Oncol Hematol. 2002; 44: 259-272. http://dx.doi.org/10.1016/S1040-8428(02)00117-8 